\title{
Mediação do erro no ensino de programação de computadores: fundamentos e aplicação da ferramenta FARMA-ALG
}

\author{
Alexander Robert Kutzke ${ }^{1}$, Alexandre Ibrahim Direne ${ }^{1}$ \\ ${ }^{1}$ Programa de Pós-Graduação em Informática - Departamento de Informática \\ Universidade Federal do Paraná (UFPR) \\ Caixa Postal 19.081 - 81.531-980 - Curitiba - PR - Brasil \\ \{alexander, alexd\}@inf.ufpr.br
}

\begin{abstract}
The computer programming is a complex, high level, activity that demands a high cognitive load. In this scenario, students make mistakes during the resolution of exercises. These errors, if not mediated culminate, ultimately, on the students' dropout. In this context, this paper aims to advance the discussions, according to a bias of Historical-Cultural Psychology, on the important role of error mediation in computer programming teaching. In order to verify such a role, the study presents details of the applicatoion of the erro mediation tool FARMA-ALG in a real teaching environment. Results obtained indicate contributions of error mediation enabled by the tool in student performance.
\end{abstract}

Resumo. A programação de computadores é uma atividade complexa, de alto nível, e que demanda uma carga cognitiva elevada. Neste cenário, alunos cometem erros durante a resolução dos exercícios. Esses erros, se não mediados, culminam, em última instância, na desistência do aluno. Nesse contexto, o presente trabalho visa avançar nas discussões, segundo um viés da Psicologia Histórico-Cultural, sobre o importante papel da mediação do erro no ensino de programação de computadores. A fim de verificar tal papel, o estudo apresenta os detalhes da aplicação da ferramenta de facilitação da mediação do erro FARMA-ALG em um ambiente real de ensino. Resultados obtidos apontam para contribuições da mediação do erro permitida pela ferramenta no desempenho dos alunos.

\section{Introdução}

O ensino de programação de computadores está em constante discussão no meio acadêmico. Esta disciplina, embora seja uma das bases para os cursos de computação, é frequentemente indicada pelos alunos como uma das mais difíceis e possui altas taxas de desistência [Robins et al. 2003].

A programação de computadores é uma atividade complexa, de alto nível, e que demanda uma carga cognitiva elevada. Este cenário complexo exige muito trabalho de professores e alunos no processo de ensino e aprendizagem. E, durante a aprendizagem, o aluno enfrenta dificuldades que, potencialmente, geram erros (respostas incorretas para exercícios). 
No ensino de disciplinas complexas, como a programação de computadores, é fundamental superar a visão negativa do erro. Segundo essa visão negativa, o erro é sinal de um problema individual do aluno, seja de sua displicência com os estudos ou sua incapacidade intelectual. Para além dessa percepção, é necessário tornar o erro parte integrante do processo de ensino e aprendizagem. É preciso, então, mediar o erro.

Mediar o erro significa torná-lo objeto de uma atividade de ensino. Problematizá-lo. Colocá-lo como elemento de um processo de desenvolvimento. Um erro apresenta uma contradição no processo de pensamento, uma inconsistência não percebida pelo aluno [Kutzke and Direne 2014]. Cabe então, ao professor, apontar essa contradição. Torná-la porta de entrada para a tomada de consciência em um nível superior. Entretanto, condições materiais pouco favoráveis enfrentadas na maioria dos ambientes de ensino podem dificultar, ou até mesmo impedir, a mediação do erro. Faz-se, assim, necessário o desenvolvimento e uso de ferramentas para auxiliar esse processo de mediação.

Nessa direção, o este trabalho apresenta dois momentos: o primeiro visa avançar na discussão, ainda nova, do papel da mediação do erro no ensino e aprendizagem de programação de computadores, a partir dos preceitos encontrados nos estudos de Vigotski e da Psicologia Histórico-Cultural. E, o segundo, avalia os impactos da mediação do erro através do uso da ferramenta FARMA-ALG em uma disciplina introdutória de programação de computadores em cursos superiores de Computação. Tal ferramenta é uma implementação para o ensino de programação de computadores do arcabouço de sistema apresentado em [Kutzke and Direne 2014]. Os testes realizados apontam para uma contribuição relevante na mediação do erro por professores e alunos.

Este artigo se organiza da seguinte maneira: a Seção 2 descreve os trabalhos relacionados ao tema de mediação do erro no ensino de programação de computadores. A Seção 3 traz a discussão do papel da mediação do erro no trabalho educativo e suas relações com as especificidades do ensino de programação. Detalhes, resultados e discussão da aplicação da ferramenta FARMA-ALG são descritos na Seção 4. Por fim, a Seção 5 apresenta as conclusões obtidas com o presente estudo e aponta trabalhos futuros.

\section{Trabalhos relacionados}

A presente seção aborda, de maneira crítica, trabalhos existentes na área de Informática Educativa relacionados à mediação do erro no ensino e aprendizagem de programação de computadores. Ao se analisarem trabalhos relacionados ao ensino de outras áreas de conhecimento, encontram-se diversas pesquisas, como [Isotani et al. 2011], que atribuem papel de importância ao erro nas atividades de ensino. Outro exemplo é a ferramenta FARMA [Marczal 2014], utilizada principalmente para o ensino de conceitos matemáticos. O estudo que apresenta esta ferramenta avança na discussão do erro e inicia um processo de favorecimento à mediação do erro. Nessa ferramenta, o aluno tem a opção de retroação ao momento e estado exatos em que cometeu um erro. Pode, assim, rever suas ações e tentar corrigi-las. O professor também tem acesso aos registros de erro armazenados.

No que diz respeito ao estudo e uso do erro (não especificamente de sua mediação) no ensino de programação de computadores, os trabalhos encontrados referem-se, em sua maioria, à análises computacionais que tratam de maneira automática os erros dos 
alunos. Grande parte dessas pesquisas se relacionam aos campos de Mineração de dados Educacionais (EDM) e de Sistemas Tutores Inteligentes (STIs).

Em EDM, as análises mais frequentes envolvem dados de taxa de erros de alunos [Rau and Scheines 2012] e o uso de ferramentas de monitoramento de estudantes [Gaudencio et al. 2013]. Entretanto, o estudo de registros de erro ainda é secundário nas pesquisas dessa área [Peña-Ayala 2014].

No que se refere a STIs, os trabalhos tratam, comumente, erros de alunos por meio de 3 processos: 1) diagnóstico, que consiste em encontrar e classificar, de maneira automática, os erros cometidos pelos alunos ([Adam and Laurent 1980], [Johnson and Soloway 1985]); 2) remediação, que é a ação, também automática, tomada pelo sistema quando um erro é capturado ([Leite et al. 2014]); e 3) classificação de erros, automática ou não ([Leite et al. 2011], [Ramos 2011]).

Conforme o levantamento realizado pelos autores deste trabalho e o número escasso de estudos encontrados, conclui-se que o tópico de pesquisa da mediação do erro especificamente para a área de programação ainda é recente. Discussões sobre a importância do tema se apresentam em estágio inicial. Apenas após o ano de 2014, os trabalhos [Kutzke and Direne 2014] e [Kutzke and Direne 2015] promoveram reflexões iniciais sobre o tema e apontam a necessidade de instrumentos para a promoção da mediação do erro nesse campo. Poucos trabalhos fornecem apoio ao professor na análise de erros de alunos e em sua consequente mediação. Em geral, apresentam processos automáticos e análises estatísticas sem a participação do professor, o que impede um estudo mais profundo das causas concretas do erro. Entende-se, assim, que existe um espaço novo e amplo de pesquisa no estudo da mediação do erro no ensino de programação de computadores. É preciso, então, avançar nas discussões do tema e avaliar as primeiras soluções apresentadas, como em [Kutzke and Direne 2015].

\section{Mediação do erro no ensino de programação de computadores}

A presente seção faz apontamentos, a partir dos estudos de Vigotski, sobre o papel da mediação do erro na educação e dos seus impactos sob as especificidades do ensino de programação de computadores. Busca-se, com isso, um enriquecimento da discussão sobre o tema, para que novos estudos sejam fomentados.

\subsection{O papel da mediação do erro na educação}

Segundo os preceitos da Psicologia Histórico-Cultural [Vigotski 2009], a forma de ensino que se propõe a contribuir para o desenvolvimento do indivíduo é aquele que promove a formação de conceitos científicos, por meio da superação dos conceitos espontâneos. Os conceitos espontâneos (CEs), referem-se aos conceitos formados no cotidiano, por meio de necessidades diretas. São conceitos não sistematizados e que possuem pouca capacidade de abstração [Vigotski 2009]. Assim, este tipo de conceito representa uma relação imediata com objeto. A palavra, que simboliza o conceito (signo), passa a ser apenas uma representação mental dos atributos puramente empíricos do objeto, ou seja, da aparência do objeto. Em suma, a mediação através da palavra se resume a uma representação direta, simples, da realidade, não passível de reflexões mais apuradas.

Já os conceitos científicos (CCs), por sua vez, são de um tipo superior, formados através de atividades mediadas e intencionais. Conceitos desse tipo não se relacionam 
diretamente com o objeto, mas sim, pela mediação de outros conceitos [Vigotski 2009]. Nesse sentido, diferentemente dos CEs, a representação do objeto passa a ser composta pelos seus atributos essenciais (atributos não-empíricos, universais). CCs, por serem mediados por outros conceitos, compõem uma espécie de sistema de conceitos. Isto é, são sistematizados.

A divisão dos conceitos em dois tipos pode gerar um entendimento incorreto de dicotomia entre eles. Em sua obra, Vigotski deixa claro que não é possível analisar o processo de formação de conceitos científicos sem que se leve em conta a relação dialética entre ambos os tipos. Conceitos do tipo científico são produtos de uma tomada de consciência de outros conceitos, potencialmente espontâneos, que são agregados ao grupo sistematizado de conceitos. Dessa forma, não existe desenvolvimento de conceitos científicos sem a passagem por conceitos espontâneos, e que estes tenham alcançado um determinado nível a fim de que haja tal tomada de consciência.

Portanto, se um conceito não é apreendido, ou não se torna consciente, integralmente de uma só vez, implica que, durante os momentos do processo de sua formação, o conceito por vezes se estrutura sobre generalizações, de certa forma, incompletas [Vigotski 2009]. Ou seja, generalizações que potencialmente podem levar a erros durante a prática do aluno. A partir dessa concepção, pode-se afirmar que, não havendo assimilação de um conceito em sua forma acabada, o erro (enquanto expressão de conceitos espontâneos) é parte integrante e fundamental do processo de formação de conceitos científicos.

A constatação acima implica que, por ser uma componente do próprio processo de formação de conceitos científicos, por um lado, o erro não pode ser encarado com intolerância. Ou seja, rejeita-se aqui as visões negativas do erro. Já por outro lado, nota-se que o papel que o erro pode e deve desempenhar sobre o desenvolvimento de conceitos desse tipo vai para além da simples identificação do erro. Isso significa que o erro não pode ser tomado apenas como uma consequência do estágio de desenvolvimento do aluno e, então, ser ignorado. Ignorar o erro é não estar atento ao processo de formação de conceitos em sua completude. Em outras palavras, ancorando-se no princípio de intencionalidade do trabalho educativo [Saviani 2012], para que o erro não se torne apenas um passo no processo empírico de apreensão da realidade, como em uma tentativa e erro, ele deve ser mediado. Entende-se, assim, que o papel do erro - a partir de uma abordagem históricocultural e através de sua mediação por parte do professor - é provocar contradições e reorganizações subjetivas no aluno que promovam a formação de conceitos científicos e, consequentemente, o desenvolvimento psíquico pleno.

\subsection{Especificidades do ensino de programação de computadores}

Durante o ensino de conhecimentos que demandam pensamento abstrato e reflexivo, como a programação de computadores, a mediação do erro se torna ainda mais necessária. A Programação de computadores é uma atividade complexa, de alto nível, e que demanda uma carga cognitiva elevada. Tal atividade caracteriza-se pelo processo de "concretização" de um algoritmo através de uma linguagem de programação com objetivo final na resolução de uma tarefa ou problema.

O tipo de pensamento capaz de produzir uma atividade de alta abstração e complexidade, como demandado pela atividade de programação, é caracterizado como "pen- 
samento abstrato ou lógico-discursivo" [Martins 2013]. A forma mais desenvolvida do pensamento, a qual apoia-se em conceitos e raciocínios abstratos operando, fundamentalmente por mediação.

Além de seus signos e operações próprias (estruturas de repetição, controle de fluxo, gerenciamento de memória, etc.) a aprendizagem da programação de computadores está ligada, também, ao domínio de uma linguagem de programação. As etapas de resolução de um problema, da execução de uma tarefa, devem ser transcritas para essa linguagem, seguindo seu alto rigor léxico, sintático e semântico.

Concluí-se assim, que a aprendizagem da atividade de programação compreende dois componentes inter-relacionados: a apropriação de signos, os quais devem exercer o papel de ferramentas psíquicas na obtenção do objetivo da atividade; e a capacidade de transcrever em uma linguagem de programação o produto gerado pela atividade mental.

Através da análise do processo envolvido na atividade de programação, pode-se afirmar que seu objeto, seu alvo, é de uma "segunda ordem". Pois, durante o processo da atividade, não basta encontrar a solução de um problema, mas sim definir passos, um algoritmo, para que todas as instâncias deste problema possam ser resolvidas. Carateriza-se um processo genuíno de generalização. Assim, não é o problema em si (primeira ordem) que se torna objeto da atividade, mas sim o processo de sua resolução (segunda ordem). Isso, por si só, já determina que a atividade de programação tem um caráter profundamente reflexivo. Esta caracterização se torna ainda mais efetiva quando se adiciona o fato de que o produto dessa atividade não é "materializável em sua plenitude".

Em outras palavras, tem-se por produto da atividade de programação a resolução de uma tarefa através da execução do código escrito em linguagem de programação e não o código em si. Sendo assim, a execução do código por um computador, representa mais um nível de indireção na objetivação da atividade. Em suma, a atividade de programação tem por objeto o processo de resolução de um problema e é objetivada na forma de código. Este, por sua vez, só se torna instrumento para outra atividade (resolução de uma instância de um problema) quando executado por um computador ou máquina semelhante. Dessa forma, para alcançar seu objetivo final, a atividade de programação passa por várias fases de abstração e de processos reflexivos e seu aprendizado depende da tomada de consciência de todo esse processo por parte do aluno.

Para amparar a complexidade de tal atividade, o aluno que aprende a programar precisa se apropriar de signos e operações de uma maneira superior. Precisa, assim, formar conceitos científicos. Só assim os conceitos se tornarão instrumentos psicológicos, estruturas complexas que reorganizam o pensamento e que agem reflexivamente. Desta forma, em uma relação dialética, possibilitam o desenvolvimento de funções psíquicas superiores as quais, por sua vez, são a base para o pensamento abstrato, necessário para a programação de computadores.

Todavia, para que a mediação do erro no ensino de programação de computadores seja efetivada, é necessário que uma série de condições adversas sejam superadas [Kutzke and Direne 2014]. Exemplos dessas condições são: número elevado de alunos e, consequentemente, de respostas a serem analisadas, falta de armazenamento dos registros de resposta, e dificuldades de acesso a tais registros, entre outros. Assim, considera-se que o uso de ferramentas que promovam a mediação do erro durante o ensino de programação 
V Congresso Brasileiro de Informática na Educação (CBIE 2016)

Anais dos Workshops do V Congresso Brasileiro de Informática na Educação (CBIE 2016)

pode favorecer a aprendizagem dos alunos.

Nesse contexto, a próxima seção descreve detalhes da aplicação da ferramenta FARMA-ALG em disciplinas introdutórias de programação em cursos superiores de Computação e os resultados obtidos. Procura-se, a partir, desse experimento, encontrar indícios que corroborem tanto com a visão da importância da mediação do erro quanto com a contribuição da ferramenta analisada para esse processo de mediação.

\section{Aplicação da ferramenta FARMA-ALG e resultados}

O FARMA-ALG [Kutzke and Direne 2015] é uma aplicação Web que visa a promoção da mediação do erro no ensino de programação de computadores. É a implementação para o domínio de programação do arcabouço de sistema apresentado em [Kutzke and Direne 2014]. Este arcabouço visa promover a mediação do erro por meio da visualização e manipulação de registros de respostas e de suas relações. Com este instrumento, permite-se ao professor uma análise do processo de formação de conceitos do aluno de uma maneira mais concreta e sintética em detrimento de uma visão imediatista e empírica.

O experimento realizado tem o objetivo de avaliar os impactos alcançados através da promoção da mediação do erro, disponibilizada pela ferramenta FARMA-ALG, no desempenho dos alunos. Assim, duas turmas de uma disciplina introdutória de programação de computadores foram observadas. Os alunos de uma das turmas tiveram acesso à ferramenta, enquanto a outra utilizou os recursos padrões já aplicados historicamente na disciplina (listas de exercícios e monitoria presencial). Ao final do curso, as notas obtidas pelos alunos das duas turmas no decorrer do semestre foram comparadas em busca de indícios de que a ferramenta atuasse de maneira positiva no aprendizado dos estudantes, resultando, inclusive, em diferenças em seus desempenhos. Os dados obtidos foram analisados através da aplicação do teste estatístico não paramétrico Wilcoxon-Mann-Whitney. Detalhes do experimento e das conclusões obtidas são descritos a seguir.

\subsection{Detalhes do experimento}

O experimento em questão foi realizado durante o primeiro semestre de 2015 , com os alunos da disciplina de Algoritmos e Estruturas de Dados 1, dos cursos de Bacharelado em Ciência da Computação e Informática Biomédica da Universidade Federal do Paraná. Essa disciplina compõe a matriz curricular do primeiro semestre de ambos os cursos.

Os alunos dos dois cursos inscritos nas disciplina foram agrupados dando origem a duas turmas mistas. A primeira, turma $\mathbf{A}$, iniciou o semestre com 59 alunos, enquanto a turma $\mathbf{B}$ foi inicialmente composta por 57 estudantes. Para o experimento, a turma A foi nomeada Grupo experimental (GE), para a qual seria disponibilizado o acesso à ferramenta FARMA-ALG. A turma B, por sua vez, foi identificada como Grupo controle (GC), cujos alunos não fariam uso da ferramenta. Ambas os grupos receberam o mesmo conteúdo durante o semestre e realizaram as mesmas avaliações.

No decorrer do curso, os alunos receberam 7 listas de exercícios, as quais deveriam ser entregues em datas previstas. As listas eram compostas por exercícios de programação de computadores para a linguagem PASCAL. Os alunos do GC resolveram as listas sem o auxílio de sistemas adicionais, ao passo que os integrantes do GE utilizaram a ferramenta FARMA-ALG como apoio para a resolução, entrega e correção de listas de exercícios. 
V Congresso Brasileiro de Informática na Educação (CBIE 2016)

Anais dos Workshops do V Congresso Brasileiro de Informática na Educação (CBIE 2016)

A avaliação da disciplina foi composta pela aplicação de 3 provas, sendo computada, ao término do semestre, a média final dos alunos. A Tabela 1 exibe as médias obtidas por cada grupo nas diferentes avaliações. Para a verificação dos impactos da ferramenta e da consequente mediação do erro, foram definidos dois testes: a primeira prova realizada foi tomada como pré-teste, utilizado para apontar se os dois grupos apresentavam distribuições semelhantes de desempenho ao início da disciplina; a média final de cada aluno foi tomada como pós-teste, para analisar a evolução do desempenhos dos alunos durante o estudo.

\begin{tabular}{ccccc}
\hline Grupo & Prova 1 (pré-teste) & Prova 2 & Prova 3 & Média Final (pós-teste) \\
\hline Controle & $\mathbf{5 8 , 3 3}$ & $\mathbf{5 8 , 0 0}$ & $\mathbf{5 4 , 2 9}$ & $\mathbf{5 7 , 4 3}$ \\
Experimental & $\mathbf{5 1 , 4 2}$ & $\mathbf{6 0 , 5 0}$ & $\mathbf{5 7 , 7 9}$ & $\mathbf{5 6 , 5 7}$ \\
Desvio padrão (GC) & 29,23 & 28,50 & 32,36 & 25,62 \\
Desvio padrão (GE) & 21,25 & 20,38 & 25,52 & 19,59 \\
\hline
\end{tabular}

Tabela 1. Tabela de médias das notas obtidas por cada grupo nas provas aplicadas.

Quatro verificações estatísticas foram realizadas. A primeira, relacionou o préteste de ambos os grupos para apontar se, inicialmente, os grupos formavam populações uniformes, com desempenhos similares. O resultado indicou que o desempenho dos grupos foi similar no pré-teste $(\mathrm{p}-\mathrm{val}$ or $=0.0892>0.05)$. A segunda e a terceira verificação visaram indicar se houve alterações significativas entre o desempenho do préteste e do pós-teste para cada grupo. Para o GC, não houve indícios estatísticos de que o desempenho do grupo mudou ( $\mathrm{p}$-valor $=0.1746>0.05)$. Em oposição, para o GE, a análise indicou que, estatisticamente, o desempenho do grupo foi modificado $(p-v a l o r=0.000005581<0.05)$. A quarta verificação, por sua vez, buscou definir se houve diferenças significativas nos desempenhos dos grupos para o pós-teste. Segundo a análise realizada, não existem, estatisticamente, indicações de diferenças entre o desempenhos dos dois grupos no pós-teste ( $\mathrm{p}$-valor $=0.3779>0.05)$. A Figura 1, apresenta a comparação dos resultados obtidos para os grupo GC e GE.

Além da análise de desempenho dos alunos, durante o experimento, uma série de dados de uso da ferramenta FARMA-ALG foram coletados, como número de visualização de registros de respostas, mensagens enviadas, entre outros. Alguns dos dados coletados são expostos na Tabela 2. Busca-se, a partir desses dados, o estudo do uso da ferramenta durante a disciplina.

\subsection{Discussão dos resultados}

A conclusão obtida através das análises apresentadas é a de que não existem razões estatísticas para afirmar a existência de diferenças significativas entre as turmas que utilizaram e não utilizaram a ferramenta FARMA-ALG ao serem consideradas as medias finais obtidas pelos alunos. Entretanto, existem diferenças estatisticamente relevantes entre os desempenhos inicial e final do GE. Tais diferenças indicam melhoras no desempenho dos alunos.

A partir da análise das médias obtidas pelo GE nas provas 2 e 3, é possível observar que essas foram maiores do que a da primeira prova. $\mathrm{O}$ mesmo não pode ser observado no GC, para o qual houve uma queda entre a média da primeira prova e as médias da terceira prova e da média final. Portanto, segundo um viés empírico, pode-se apontar a 
V Congresso Brasileiro de Informática na Educação (CBIE 2016)

Anais dos Workshops do V Congresso Brasileiro de Informática na Educação (CBIE 2016)

\begin{tabular}{cccc}
\hline Tipo & Professor & Alunos & Total \\
\hline Mensagens enviadas & 12 & 0 & 12 \\
Visualização de mensagem & 22 & 643 & 665 \\
Visualização rápida de resposta & 575 & 359 & 934 \\
Visualização completa de resposta & 305 & 373 & 678 \\
Cliques no botão "fazer nova tentativa" & - & 91 & 91 \\
Visualização de linha do tempo & 31 & 12 & 43 \\
Visualização de estatísticas do aluno & 17 & 2091 & 2108 \\
Visualização de estatísticas de O.A. & 15 & 511 & 526 \\
Visualização de estatísticas de Questão & 180 & 365 & 545 \\
Respostas submetidas & - & 3693 & \\
Quantidade de usuários & 1 & 59 & \\
\hline
\end{tabular}

Tabela 2. Dados de uso da ferramenta FARMA-ALG coletados durante o experimento

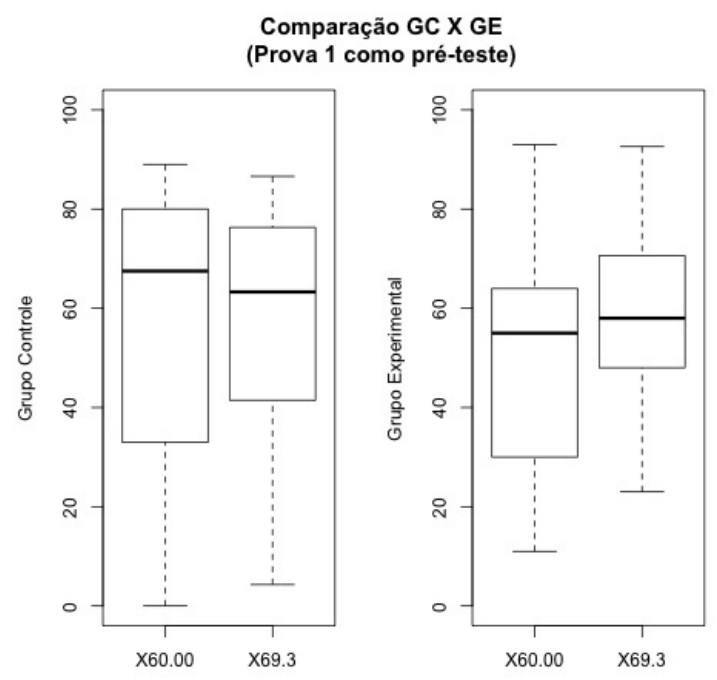

Figura 1. Comparação das notas obtidas (prova 1 e média final) pelos grupos GC e GE.

hipótese de que a ferramenta FARMA-ALG contribuiu para a melhora nas notas do GE durante as duas últimas avaliações (período em que o FARMA-ALG foi utilizado com mais intensidade). Vale ressaltar, também, que existe um salto no nível de dificuldade exibido entre as provas 1 e 2 . Ao que parece, o GE foi capaz de absorver o aumento de complexidade do conteúdo da disciplina mais satisfatoriamente.

O estudo dos dados de uso extraídos da ferramenta FARMA-ALG proporciona conclusões que apontam para a promoção da mediação do erro. Especificamente, o alto número de visualização de respostas e de visualização de mensagens indicam para a compreensão de que a mediação realizada pelo professor da disciplina através da ferramenta promoveu a reflexão dos alunos. As semanas em que houve mensagens enviadas foram as que apresentaram o maior número de visualização de respostas. Fica evidente a correlação positiva entre a mediação do professor e a participação dos alunos. A quantidade de cliques no botão "fazer nova tentativa", o qual é exibido na visualização completa de respostas, também sugere que, por várias vezes, alunos realizaram novas tentativas de resposta ao observarem suas respostas e comentários do professor. 
Além disso, mais duas conclusões podem ser formuladas ao serem considerados os dados de desempenho coletados nos últimos seis anos (11 semestres) da mesma disciplina de programação. Em primeiro lugar, apenas 18,8\% das turmas analisadas apresentaram melhora de desempenho na segunda prova em comparação com a primeira avaliação. Em segundo lugar, o GE teve a terceira menor taxa de desistência (GE: 25,42\%; GC: $38,59 \%$ ) dentre os registros observados. Até mesmo alunos do GE com baixo desempenho continuaram até a conclusão da disciplina, comportamento não observado no GC. Ambos os fatores apontam para um entendimento de que a mediação realizada através da ferramenta proporcionou melhores condições de aprendizagem para os alunos.

De forma geral, a despeito da análise estatística não ter indicado diferenças entre as notas dos pós-testes dos grupos GC e GE, assinalou-se uma melhora de desempenho obtida pelo GE. Ademais, a análise empírica dos dados produz indícios para a confirmação de que a ferramenta FARMA-ALG tenha contribuído positivamente no desempenho dos alunos por meio da mediação realizada. Certamente, novos testes devem ser realizados para que a confirmação dessa hipótese seja alcançada. Porém, não se podem descartar os sinais positivos levantados pelo presente experimento.

\section{Conclusão}

No presente trabalho, foi exposta a necessidade da mediação do erro para uma educação que promova o desenvolvimento dos alunos, especialmente em áreas complexas como a programação de computadores. Através da revisão bibliográfica, concluiu-se que o estudo do tema ainda se encontra em estágio inicial. Procurou-se, assim, através da discussão sobre o papel da mediação do erro no desenvolvimento de conceito científicos, avançar na discussão, destacando seus principais fundamentos. Definiu-se a importância da mediação do erro no ensino de programação de computadores. Ao final, com o objetivo de verificar os impactos da mediação do erro, foram expostos detalhes e os resultados da aplicação da ferramenta FARMA-ALG em turmas de disciplinas introdutórias de programação. Por meio da discussão dos resultados obtidos, faram levantadas indicações de que a mediação promovida pela ferramenta produziu impactos positivos na aprendizagem dos alunos.

Embora o estudo apresentado tenha exibido conclusões iniciais sobre o tema, avanços para a importante discussão da mediação do erro no ensino de programação de computadores foram realizados. A efetiva aplicação de ferramentas como o FARMAALG em ambientes reais de ensino e a posterior análise dos resultados contribui para a evolução do tema, o que fomenta novas pesquisas. Assim, para o prosseguimento do estudo exposto, trabalhos futuros incluem evolução na discussão da mediação do erro no ensino de programação de computadores e novas aplicações da ferramenta FARMA-ALG para que se possa verificar com mais objetividade suas contribuições, em especial, sob a prática docente.

\section{Referências}

Adam, A. and Laurent, J.-P. (1980). Laura, a system to debug student programs. Artificial Intelligence, 15(1-2):75-122.

Gaudencio, M., Wanderley, L. F., Lemos, F. W., de Araújo, E. C., Figueiredo, J. C., and Guerrero, D. D. (2013). Eu sei o que vocês fizeram (agora e) na aula passada: o tstview 
V Congresso Brasileiro de Informática na Educação (CBIE 2016)

Anais dos Workshops do V Congresso Brasileiro de Informática na Educação (CBIE 2016)

no acompanhamento de exercícios de programação. In Anais do Simpósio Brasileiro de Informática na Educação, volume 24.

Isotani, S., Adams, D., Mayer, R. E., Durkin, K., Rittle-Johnson, B., and McLaren, B. M. (2011). Can erroneous examples help middle-school students learn decimals? In Towards Ubiquitous Learning, pages 181-195. Springer.

Johnson, W. and Soloway, E. (1985). Proust: Knowledge-based program understanding. Software Engineering, IEEE Transactions on, SE-11(3):267-275.

Kutzke, A. R. and Direne, A. I. (2014). Mediação do erro na educação: um arcabouço de sistema para a instrumentalização de professores e alunos. In Anais do Simpósio Brasileiro de Informática na Educação, volume 25.

Kutzke, A. R. and Direne, A. I. (2015). FARMA-ALG: An Application for Error Mediation in Computer Programming Skill Acquisition, pages 690-693. Springer International Publishing, Cham.

Leite, M. D., Marczal, D., Pimentel, A. R., and Direne, A. I. (2014). A conceptual framework to use remediation of errors based on multiple external remediation applied to learning objects. Journal of Technology and Science Education, 4(3):155-166.

Leite, M. D., Pimentel, A. R., and Oliveira, F. D. (2011). Um estudo sobre classificação de erros: uma proposta aplicada a objetos de aprendizagem. In Anais do Simpósio Brasileiro de Informática na Educação, volume 1.

Marczal, D. (2014). FARMA: Uma ferramenta de autoria para objetos de aprendizagem de conceitos matemáticos. Doutorado em informática, Universidade Federal do Paraná - Departamento de Informática, Curitiba, PR, Brasil.

Martins, L. M. (2013). O Desenvolvimento do Psiquismo e a Educação Escolar: contribuições à luz da psicologia histórico-cultural e da pedagogia histórico-crítica. Autores Associados, Campinas, SP, 1 edition.

Peña-Ayala, A. (2014). Educational data mining: A survey and a data mining-based analysis of recent works. Expert Systems with Applications, 41(4, Part 1):1432 - 1462.

Ramos, G. S. (2011). Detecção e remediação de erros na generalização de conceitos matemáticos por meio de sistemas tutores inteligentes. Mestrado, Universidade Federal do Paraná - Departamento de Informática, Curitiba, PR, Brasil.

Rau, M. and Scheines, R. (2012). Searching for variables and models to investigate mediators of learning from multiple representations. In The 5th International Conference on Educational Data Mining (EDM 2012), pages 110-117.

Robins, A., Rountree, J., and Rountree, N. (2003). Learning and teaching programming: A review and discussion. Computer Science Education, 13(2):137-172.

Saviani, D. (2012). Pedagogia Histórico-Crítica. Primeiras Aproximações. Autores Associados, Campinas, SP, 11 edition.

Vigotski, L. S. (2009). A construção do pensamento e da linguagem. Martins Fontes, São Paulo, 2 ed. edition. 